\title{
COMMENT \\ Rigorous and consistent evaluation of diagnostic tests in children: another unmet need
}

\author{
Christine S. Caldwell ${ }^{1}$, Scott C. Denne ${ }^{2}$ and On behalf of the Pediatric Policy Council \\ Pediatric Research (2020) 88:524-525; https://doi.org/10.1038/s41390-020-01110-0
}

Accurate diagnosis is fundamental to medical practice. While there is often more focus on therapeutics, the importance of diagnostics has been highlighted by the COVID-19 pandemic. With the concerns about both false-negative rates for the COVID-19 PCR test, and false-positive rates for the COVID-19 antibody test, both physicians and the public are more acutely aware of the importance of accurate diagnostic testing., ${ }^{1,2}$

In this issue of Pediatric Research, Rowland et al. ${ }^{3}$ examined the repeatability of transient elastography (TE), a widely used method to diagnose liver disease in children. The study demonstrated that despite its widespread use, TE does not have adequate precision for use in children. Similar to the situation for COVID-19 testing, this study demonstrates the need for rigorous evaluation and a complete understanding of diagnostic techniques before they are fully employed in clinical practice. Furthermore, diagnostics, like therapeutics, are most often developed for and tested in adults. Additional evaluation in children may be required to establish proper efficacy.

While there is a general understanding of the drug evaluation process, the evaluation for diagnostic tests and techniques can be confusing and is not well understood by either the public or physicians. The regulatory pathway falls under the category of devices and is significantly different than the process for drug approvals.

The Food and Drug Administration (FDA) is responsible for approving or clearing drugs, biologics, and medical devices for marketing. Drugs, biologics, and devices are evaluated by different centers within the FDA under different regulations, creating different pathways for product evaluation. Drugs and biologics are regulated by the Center for Drug Evaluation and Research and Center for Biologics Evaluation and Research, respectively, while devices are regulated by the Center for Devices and Radiological Health. Devices are classified by risk level, from Class I (lowest risk) to Class III (highest risk). ${ }^{4}$ Diagnostic tests, such as in vitro diagnostic and imaging devices, are typically Class I or Class II devices. To be cleared for marketing, Class I and II devices require a premarket notification (or a $510(\mathrm{k})$ ). A $510(\mathrm{k})$ submission must demonstrate that the proposed device is as "safe and effective, that is, substantially equivalent, to a legally marketed device". ${ }^{5} \mathrm{~A}$ legally marketed device to which a new device is compared is often called a predicate device. Class III devices, on the other hand, require a more stringent review process, including laboratory and clinical data demonstrating that the device is safe and effective, most commonly through a premarket approval (PMA) application. ${ }^{6}$
Unlike new drug applications, ${ }^{7}$ new device applications are not required to include clinical study data from pediatric patients. PMAs and other new device submissions must include any readily available information about affected pediatric subpopulations as well as the number of affected pediatric patients, ${ }^{8}$ but this falls short of requiring data from clinical studies in pediatrics. As a result, many devices (including diagnostic tests) are not designed with children in mind. Pediatricians use devices approved for adults "off-label" in children, meaning the device is used for an indication that is not part of the FDA-approved label. The FDA considers off-label use to be the practice of medicine, something the FDA does not regulate. There is an unspoken understanding that market clearance means devices can be used off-label for different populations.

While others have rightly pointed out that off-label use of devices and diagnostic tests in pediatric patients is necessary and may be appropriate in some circumstances, ${ }^{9}$ those recommendations relate to higher risk devices (Class III). However, most diagnostic devices are Class I and II, and FDA clearance for marketing is based on the claim that the new device is as safe and effective as a substantially equivalent predicate device. This pathway is most commonly used, although the de novo pathway is an option when there is no predicate device.

The highest evidentiary standard for most diagnostic devices, then, is a comparison to a predicate device "rather than an independent demonstration of the new device's safety and effectiveness" ${ }^{10}$ There is no specific requirement that diagnostic devices demonstrate sensitivity and specificity in children. With this standard applying to Class I and II devices, it is not surprising that device companies seek FDA clearance to market their devices without mandate or incentive to collect data on use in children. The FDA is attempting to address the problems faced in pediatric device development through initiatives, such as the Pediatric Device Consortia Grants Program, System of Hospitals for Innovation in Pediatrics (SHIP), and pediatric expertise on advisory panels. ${ }^{11-13}$ Despite these initiatives, many diagnostic tests cleared by the FDA lack sufficient evidence for use in children. Because the prevalence of many diseases is lower in children than adults, relying on adult derived specificity and sensitivity assessments may be misleading for pediatric clinicians.

While the pediatric community has made great strides to ensure proper evaluation of drugs in children, ensuring the same quality evaluation of devices, including diagnostic tests, remains elusive. This gap requires pediatricians to seek out and understand the


University, Indianapolis, IN, USA

Correspondence: Scott C. Denne (sdenne@iu.edu)

A list of members of the Pediatric Policy Council (PPC) appears below acknowledgement.
}

Received: 25 June 2020 Revised: 31 July 2020 Accepted: 7 August 2020

Published online: 6 September 2020 
limitations of diagnostic tests used in children, and the academic community to undertake rigorous studies of diagnostics, as Rowland and colleagues have done. Given the wide range and number of diagnostic tests, this situation is neither sustainable nor realistic for pediatricians or the academic community.

To be sure, there are substantial barriers that must be addressed to make sure that diagnostic tests are consistently studied in children. Diagnostic tests may only be used in a small number of children, and there are significant development and regulatory costs with limited financial return. ${ }^{14}$ In addition, smaller companies with fewer resources predominate in the medical device industry, and devices and tests are often developed in an iterative process by refining devices and tests over time, with a new design replacing the old over a relatively short time. ${ }^{15}$ This process makes a key incentive for the study of drugs in childrenan extended period of market exclusivity-less useful for device companies.

The challenges of making properly evaluated devices and diagnostic tests available to children have long been recognized, and in 2007, Congress passed the Pediatric Device Safety and Improvement Act. $^{16}$ This law facilitated tracking of pediatric approvals and strengthened post-market surveillance of pediatric devices, but did not include any type of incentives and mandates that have transformed pediatric drug availability. This act has had only modest success, and it falls far short of the record of achievement for drugs in children. ${ }^{15}$

It is time for the pediatric community, along with the FDA and the device industry, to renew efforts to better provide appropriately evaluated devices and diagnostic tests for children. This will require seriously considering incentives and/or funding for pediatric trials, improving insurance coverage to reimburse for device use, and creative approaches to the evaluation process in children. ${ }^{14}$ In the meantime, the FDA should work to better communicate the device and diagnostic test regulatory process to the public and physicians, and make clear to pediatricians to what extent specific diagnostic tests and devices have and have not been evaluated in children.

\section{ACKNOWLEDGEMENTS}

This work was supported in part by the Indiana Clinical and Translational Sciences Institute funded, and in part by Award Number UL1TR002529 from the National Institutes of Health, National Center for Advancing Translational Sciences, and Clinical and Translational Sciences Award.

\section{PEDIATRIC POLICY COUNCIL (PPC)}

Scott C. Denne, M.D., Chair, Pediatric Policy Council; Mona Patel, M.D., Representative to the PPC from the Academic Pediatric Association; Jean Raphael, M.D., M.P.H. Representative to the PPC from the Academic Pediatric Association; Jonathan Davis, M.D., Representative to the PPC from the American Pediatric Society; DeWayne Pursley, M.D., M.P.H., Representative to the PPC from the American Pediatric Society;
Tina Cheng, M.D., M.P.H., Representative to the PPC from the Association of Medical School Pediatric Department Chairs; Michael Artman, M.D., Representative to the PPC from the Association of Medical School Pediatric Department Chairs; Shetal Shah, M.D. Representative to the PPC from the Society for Pediatric Research; Joyce Javier, M.D. M.P.H., M.S., Representative to the PPC from the Society for Pediatric Research.

\section{ADDITIONAL INFORMATION}

Competing interests: The authors declare no competing interests.

Publisher's note Springer Nature remains neutral with regard to jurisdictional claims in published maps and institutional affiliations.

\section{REFERENCES}

1. Basu, A. et al. Performance of Abbott ID NOW COVID-19 rapid nucleic acid amplification test in nasopharyngeal swabs transported in viral media and dry nasal swabs, in a New York City academic institution. J. Clin. Microbiol. https://doi. org/10.1128/JCM.01136-20 (2020).

2. Hwang, T. J., Kesselheim, A. S. \& Bourgeois, F. T. Postmarketing trials and pediatric device approvals. Pediatrics 133, e1197-e1202 (2014).

3. Rowland, M. et al. Repeatability of transient elastography in children. Pediatr. Res. https://doi.org/10.1038/s41390-020-0916-4 (2020).

4. FDA. Classify your medical device. https://www.fda.gov/medical-devices/ overview-device-regulation/classify-your-medical-device (2020).

5. FDA. Premarket notification. https://www.fda.gov/medical-devices/premarketsubmissions/premarket-notification-510k (2020).

6. FDA. FDA premarket approval (PMA). https://www.fda.gov/medical-devices/ premarket-submissions/premarket-approval-pma (2020).

7. PREA. Pediatric Research Equity Act of 2003. https://www.congress.gov/108/ plaws/publ155/PLAW-108publ155.pdf (2020).

8. 21 USC § 360e-1. https://www.govinfo.gov/content/pkg/USCODE-2011-title21/ html/USCODE-2011-title21-chap9-subchapV-partA-sec360e-1.htm (2020).

9. American Academy of Pediatrics. Off label use of medical devices in children. Pediatrics 139, e20163439 (2017).

10. US FDA. FDA 2014. The 510(k) Program: Evaluating Substantial Equivalence in Premarket Notifications [510(k)]: Guidance for Industry and Food and Drug Administration Staff. https://www.fda.gov/regulatory-information/search-fdaguidance-documents/510k-program-evaluating-substantial-equivalencepremarket-notifications-510k (2020).

11. US FDA. Pediatric Device Consortia Grants Program. https://www.fda.gov/ industry/developing-products-rare-diseases-conditions/pediatric-deviceconsortia-grants-program (2020).

12. US FDA. Rare disease day 2020: FDA continues important work on treatment for rare diseases. https://www.fda.gov/news-events/fda-voices/rare-disease-day2020-fda-continues-important-work-treatments-rare-diseases (2020).

13. US FDA. Pediatric Expertise for Advisory Panels-Guidance for Industry and FDA Staff. https://www.fda.gov/regulatory-information/search-fda-guidance-documents/ pediatric-expertise-advisory-panels-guidance-industry-and-fda-staff (2020).

14. AAP News and Journals. AAP brings need for pediatric medical devices to forefront. https://www.aappublications.org/news/2018/08/17/fdadevices081718 (2020).

15. Ulrich, L. C., Joseph, F. D., Lewis, D. Y. \& Koenig, R. L. FDA's pediatric device consortia: national program fosters pediatric medical device development. Pediatrics 131, 981-985 (2013).

16. FDA. Food and Drug Administration Amendments Act of 2007. https://www. congress.gov/bill/110th-congress/house-bill/3580 (2020). 